
\title{
25 Research Soure \\ Enzyme JARID1B could be new target for fighting spread of colorectal cancer
}

Da Huang

Fan Xiao

Haibin Hao

Fuzhou Hua

Zhenzhong Luo

Zhaoxia Huang

Qing Li

Sha Chen

Xiuzhi Cheng

Xinyue Zhang

Weilan Fang

Xiaoyun Hu

Fanrong Liu

\section{Video Byte}

Keywords: Cell Communication and Signaling, colorectal cancer, cell proliferation, JARID1B, CDX2, H3K4me3, Wnt/ $\beta$-catenin, Jumonji AT-rich interactive domain 1B, CRC, western blotting, qRT-PCR, ChIP assay, tumor, demethylation, proliferation, survival, pathogenesis, metastasis, epigenetic regulation, tumorigenesis

Posted Date: November 12th, 2020

DOl: https://doi.org/10.21203/rs.3.rs-107378/v1

License: (9) This work is licensed under a Creative Commons Attribution 4.0 International License. Read Full License 


\section{Abstract}

Colorectal cancer is the most common cancer worldwide and among the top 3 causes of cancer-related death in men and women. Despite advances in diagnosing and treating colorectal cancer prognosis remains poor because of persistent mechanisms of tumor proliferation. A new study has zeroed in on one protein that could be behind some of these mechanisms of colorectal cancer spread. JARID1B is a demethylase enzyme encoded by the gene KDM5B and has been implicated in the development of several cancers, including breast, prostate, and liver cancer. Researchers found that JARID1B was significantly upregulated in colorectal cancer tissue versus adjacent normal tissue. In patients with colorectal cancer, high JARID1B expression was associated with poor overall survival. Experiments revealed that JARID1B promoted the spread of colorectal tumor cells through the $W n t / \beta$-catenin signaling pathway. Specifically, by inhibiting the protein CDX2. More work is needed to understand whether and how this mechanism occurs in humans and how JARID1B might be manipulated to develop new therapies for treating colorectal cancer. 